TRANS · núm. $24 \cdot 2020$

DOSIER $\cdot 145-164$

El estudio que presentamos pretende describir el panorama actual de la formación en accesibilidad en España. A los cursos de posgrado universitarios sobre audiodescripción y subtitulado para sordos que se vienen ofreciendo hasta el momento, se suma el curso de especialización en accesibilidad recientemente aprobado por el Real Decreto 94/2019, de 1 de marzo, por el que se establece el Curso de especialización en audiodescripción y subtitulación y se fijan los aspectos básicos del currículo. Una vez analizada la oferta formativa, estudiamos las competencias que se pretende que adquiera el alumnado atendiendo a la clasificación de competencias que debe adquirir el «accesibilitador» (Díaz Cintas, 2007a). Los resultados indican que la accesibilidad se encuentra presente en numerosos cursos de posgrado, aunque se aborda de manera muy heterogénea. No obstante, existe cierto consenso en cuanto a las competencias que se exige al futuro experto en accesibilidad.

PALABRAS CLAVE: Traducción audiovisual, audiodescripción, accesibilidad, competencias, formación.

\title{
La formación del audiodescriptor en España. Situación actual y retos futuros
}

Raquel Sanz-Moreno Universitat de València

\section{The Audio Describer Training in Spain: current situation and future challenges}

This study aims to describe the current panorama of accessibility training in Spain. In addition to the postgraduate university courses on audio description and subtitling for the deaf that have been offered up to now, there is also the specialization course in accessibility recently approved by Royal Decree 94/2019. Once the training programmes have been analyzed, we describe the competences that the students are expected to acquire according to the classification of competences that the "accessibilitator» must acquire (Díaz Cintas, 2007a). The results indicate that accessibility is present in many postgraduate courses, although it is approached in a very heterogeneous way. Nevertheless, there is a certain consensus regarding the competencies required for the future accessibility expert.

KEY WORDS: audiovisual translation, audio description, accessibility, competences, postgraduates programmes. 


\section{1. INTRODUCCIÓN}

La audiodescripción (AD) comenzó a ofrecerse en España en los años 90 gracias a la ingente labor de la ONCE (Organización Nacional de Ciegos de España), que se encargaba de audiodescribir todas las películas a las que tenían acceso sus afiliados. Lamentablemente, estas películas audiodescritas de innegable valor no estaban a disposición del público general y no se comercializaban, por lo que incluso la existencia de la AD era desconocida para el gran público. Los audiodescriptores de la ONCE fueron los pioneros de la AD en España, los primeros que establecieron qué y cómo debían audiodescribirse las películas y las obras teatrales (Navarrete, 1997a, 1997b). Sus recomendaciones, aún hoy en día, se siguen para elaborar guiones de AD. De hecho, la ONCE jugó un papel fundamental en la aprobación de la norma UNE AENOR 153020 de 2005 sobre «Audiodescripción para personas con discapacidad visual. Requisitos para la audiodescripción y elaboración de audioguías», cuyo fin era uniformizar la $\mathrm{AD}$ en España y establecer las directrices sobre cómo realizarla.

Sin embargo, quince años más tarde, la situación ha cambiado notablemente, y no solo respecto a la producción sino también al consumo de productos audiovisuales: la presencia de plataformas de streaming, el auge del consumo de series de televisión frente a películas, sobre todo en la gran pantalla, y en distintos soportes (ordenadores, tabletas, móviles, etc.), y el aumento de la demanda de la televisión a la carta, entre otros factores, hacen que cada vez más operadores manifiesten un interés creciente por hacer accesibles los productos audiovisuales que ofrecen mayoritariamente a través de la $\mathrm{AD}$ y de la subtitulación para sordos $(\mathrm{SpS})^{1}$. Y dado que cada vez se ofrecen más programas audiovisuales accesibles, es necesario contar con más profesionales expertos en accesibilidad audiovisual en el mercado de trabajo español. Esta es la causa principal que ha motivado al gobierno español a aprobar recientemente el Real Decreto 94/2019 de 1 de marzo, por el que se establece el curso de especialización en audiodescripción y subtitulación y se fijan los aspectos básicos del currículo, enmarcado en los ciclos formativos de grado superior de imagen y sonido. Su finalidad es dar una respuesta rápida «al aumento del consumo de contenido audiovisual y multimedia en la sociedad, aportando de este modo apoyo profesional a un nicho de mercado en crecimiento» (CESyA, 2019). No debemos olvidar, sin embargo, que la figura del audiodescriptor existe desde hace más de treinta años en España, y que su formación ha recaído, sobre todo, a lo largo de estos últimos años, y aun actualmente, en las universidades, en particular, en cursos de máster más generales sobre traducción audiovisual (TAV).

El objetivo del presente estudio es describir y analizar el panorama actual de la oferta formativa en $\mathrm{AD}$ en España a nivel de posgrado, y compararlo con el grado superior en accesibilidad aprobado recientemente por el actual gobierno español. Para ello, en un primer momento, presentaremos los trabajos previos que se han realizado sobre didáctica de la accesibilidad en nuestro país hasta el momento. Posteriormente, abordaremos la formación en $\mathrm{AD}$ que se ofrece en la actualidad en las universidades españolas

I En España, la interpretación en lengua de signos de productos audiovisuales sigue siendo una solución de accesibilidad residual que se observa, por ejemplo, en algunos documentales o series emitidos por La Sexta (grupo Atresmedia). 
y la compararemos con el currículo diseñado por el gobierno español para el curso de especialización en accesibilidad recientemente aprobado. Concluiremos con una reflexión crítica sobre la formación y competencias requeridos para los futuros «accesibilitadores» en España.

\section{TRABAJOS PREVIOS SOBRE DIDÁCTICA DE LA AD EN ESPAÑA}

Tradicionalmente, la $\mathrm{AD}$ se ha considerado una modalidad de TAV (Díaz Cintas, 2005, 2007b; Jiménez Hurtado, 2007; Maszerowska, Matamala y Orero, 2014), por lo que los estudios sobre la didáctica de la accesibilidad se han enmarcado de forma global dentro de la enseñanza de la $\mathrm{TAV}^{2}$. Es el caso de los estudios de Chaume (2003), Díaz Cintas et al. (2006), Díaz Cintas (2008) o Martínez Sierra (2008), todos ellos antecedentes del trabajo más reciente de Cerezo Merchán (2013).

En cuanto a la enseñanza más específica de la $\mathrm{AD}$, los trabajos de Matamala (2006) y Díaz Cintas (2007a) abordaban las competencias que estos autores consideraban necesarias para los futuros profesionales de la accesibilidad en España. Asimismo, Matamala y Orero (2007) publicaron el diseño de un curso sobre AD de veinte horas de duración que se iba a impartir en la Universidad Autónoma de Barcelona y en el que también se adentraban en las competencias que debía desarrollar el futuro experto en accesibilidad. Por otra parte, Badía y Matamala (2007) ofrecían un panorama de la enseñanza

\footnotetext{
2 Una parte importante de los trabajos publicados hasta ahora sobre didáctica de la TAV se centra en la utilización de las modalidades de TAV (subtitulación inter e intralingüística, doblaje, $\mathrm{AD}$, voces superpuestas, etc.) para la enseñanza de lenguas extranjeras. Sirvan como ejemplo los trabajos de Talaván (2011, 2013), Talaván y Ávila-Cabrera (2015), Calduch y Talaván (2017), Lertola $(2015,2018)$, Torralba (2017, 2018) o Alonso Pérez y Sánchez-Requena (2018).
}

en accesibilidad en España, pocos años después 147 de que entrara en vigor la Ley 51/2003, de 2 de diciembre, de igualdad de oportunidades, no discriminación y accesibilidad universal de las personas con discapacidad (LIONDAU), en un claro antecedente del trabajo que aquí presentamos. La citada ley preveía campañas de sensibilización y acciones formativas para promover y garantizar la igualdad entre ciudadanos y establecía un plazo de dos años para desarrollar un currículo formativo de «diseño para todos» en el que se incluían también «las comunicaciones y telecomunicaciones y los servicios de la sociedad de la información» (disposición adicional décima de la LIONDAU). Tal y como ya apuntaban estos autores, «no hay duda de que la formación en accesibilidad en los medios irá incrementando su presencia» (2007: 62), predicción que se ha cumplido por lo que, más de diez años después, consideramos que se hacía necesaria una revisión y actualización del estado de la cuestión de la enseñanza de la AD audiovisual en nuestro país, y más teniendo en cuenta la aprobación del real decreto 94/2019 de 1 de marzo antes citado, como veremos más adelante.

Más recientemente, las autoras Mendoza y Matamala presentan un estudio sobre la enseñanza de la $\mathrm{AD}$ en España basándose en un cuestionario a 27 encuestados. Entre las competencias más relevantes de un audiodescriptor, destacan la capacidad para la elección de la información relevante, el dominio de la lengua materna y el conocimiento de las necesidades del usuario (2018: 21). Las autoras ahondan en las competencias y habilidades del descriptor en España en un trabajo posterior y concluyen, entre otros que los audiodescriptores otorgan especial importancia a los aspectos textuales y lingüísticos. A través de un cuestionario, las habilidades identificadas se comparan con las contempladas por cursos de AD. Algunas ha- 
148 bilidades como la capacidad de improvisación o de gestionar la presión del tiempo no se contemplan por no encontrarlas necesarias por los encuestados (Mendoza y Matamala, 2020: 164). En la misma línea, Sanz-Moreno (2018) realiza un estudio exploratorio basado en datos cualitativos y cuantitativos obtenidos a través de un cuestionario a 14 audiodescriptores que trabajan actualmente en el mercado español, y en el que, entre otros, los profesionales evalúan las competencias que consideran que los descriptores en formación deben reunir para ejercer su profesión (íbid. 132-135). El conocimiento exhaustivo del idioma materno, de las otras modalidades de accesibilidad, de programas informáticos y de Internet, así como la capacidad de análisis y síntesis, y de interpretación de la información son las competencias mejor valoradas por los audiodescriptores encuestados.

\section{METODOLOGÍA}

Nuestro trabajo no parte de ninguna hipótesis en concreto, sino que nos planteamos una serie de preguntas con las que nos adentramos en el panorama actual de la didáctica de la accesibilidad en España. Nos encontramos, pues, ante un estudio exploratorio secuencial cuya naturaleza es descriptiva e interpretativa, ya que nuestra intención es acercarnos a la realidad de la formación en accesibilidad en España, describirla tal y como es, e interpretarla.

Con esa finalidad, una vez reunimos el material sobre nuestro objeto de estudio (ver apartado 3.1.), procedimos a ordenarlo para describirlo de forma sistemática y metódica. Se siguió la técnica de análisis de contenido, que podemos definir como «una técnica de investigación para la descripción objetiva, sistemática y cuantitativa del contenido manifiesto de las comunicaciones, que tiene como primer objetivo interpretarlas» (Berelson en López Noguero, 2002: 174-175). En este apartado presentamos la metodología del estudio que hemos aplicado: en un primer momento, describimos los documentos que se han analizado para la obtención de datos, es decir, el objeto de nuestro estudio, y a continuación, explicamos el procedimiento de análisis de los datos recolectados.

\subsection{Objeto de estudio: materiales analizados}

Nuestro objeto de estudio son aquellos programas de formación universitaria en los que se ofertan asignaturas de accesibilidad audiovisual (tanto títulos oficiales como propios, de universidades públicas y privadas, y de institutos dependientes de universidades). Para determinar los cursos de posgrado oficiales que se imparten actualmente en universidades españolas sobre traducción, hemos consultado el Registro de Universidades, Centros y Títulos (RUCT), dependiente del Ministerio de Ciencia, Innovación y Universidades. En esta valiosa base de datos se incluyen los enlaces a los Boletines Oficiales del Estado que publican su aprobación, su renovación y los programas de estudio de cada máster, que también han sido consultados y revisados para este estudio. También se han consultado las distintas páginas web de las universidades o institutos que ofertan másteres en traducción durante el curso 2018-2019.

Se identificaron en total 29 estudios de posgrado (oficiales o propios) sobre traducción (general y especializada) actualmente en vigor en España, de universidades tanto públicas como privadas, pero obviamente no en todos se ofertaban asignaturas sobre accesibilidad. Se descartaron para su análisis aquellos másteres que no incluían ninguna asignatura sobre accesibi- 
lidad o sobre TAV, así como aquellos sobre modalidades de traducción especializada (médica, institucional o jurídica, interpretación en los servicios públicos o de conferencias, etc.) o sobre investigación. Como veremos más adelante, solo se oferta un curso específico sobre accesibilidad; la gran mayoría de cursos de posgrado que ofertan algunas asignaturas sobre AD y SpS son másteres especializados en TAV. También encontramos, de forma más excepcional, posgrados de traducción general que abordan de manera puntual la accesibilidad (ver apartado 4.1).

Una vez clasificados todos los posgrados, se analizaron los respectivos programas de estudios oficiales publicados en el Boletín Oficial del Estado, así como en las páginas web de cada máster. Finalmente, también se analizaron las guías docentes de las asignaturas correspondientes al curso 2018-2019. Este análisis pormenorizado perseguía determinar los contenidos, la modalidad de docencia (teórica, práctica o mixta) y las competencias que se pretendían adquirir al cursar estas asignaturas.

Además, analizamos el currículo del grado superior sobre el curso de especialización en AD y SpS publicado en el Real Decreto 94/2019. A pesar de que no se ha desarrollado aún, el texto legal ofrece información sobre las asignaturas que componen el curso, la duración y las competencias que se pretende que adquiera el alumnado.

En resumen, los datos que se han analizado para realizar este estudio provienen de las siguientes fuentes de información:

- Planes de estudios de posgrados (oficiales, propios) de universidades españolas publicados en el BOE.

- Páginas web oficiales de los programas de posgrado.

- Guías docentes de asignaturas sobre accesibilidad y TAV.
- Comunicaciones personales con responsables de los distintos estudios y profesores de diferentes asignaturas ${ }^{3}$.

- Currículo del Curso de especialización en $\mathrm{AD}$ y $\mathrm{SpS}$ establecido por el real decreto 94/2019.

\subsection{Procedimiento de análisis}

Para determinar las competencias que se contemplan en los distintos programas de posgrado y en el nuevo curso de especialista en accesibilidad, hemos analizado las guías docentes publicadas en las respectivas páginas web (o que nos han hecho llegar los profesores o coordinadores de las asignaturas) en las que se especifican las competencias que se pretende que el alumnado adquiera, así como el currículo (concretamente el artículo 6 del real decreto 94/2019) del grado superior. Hemos partido del listado de competencias que proponía Díaz-Cintas en 2007 en lo que respecta a una preparación de calidad en accesibilidad y hemos identificado aquellas competencias que están incluidas (de forma más o menos explícita) en las guías docentes y en el currículo.

Las competencias de las que partimos están clasificadas en cuatro grandes bloques: competencias lingüísticas, competencias temáticas, competencias informáticas y aplicadas y competencias personales. Cada bloque contiene diferentes sub-competencias, la mayoría sobre accesibilidad en general, aunque algunas son específicas de AD o de SpS.

En primer lugar, comprobamos si las competencias de la Tabla 1 aparecían en las guías do-

\footnotetext{
3 Agradecemos a los coordinadores y profesores de estudios de posgrado que nos han atendido y facilitado información sobre los distintos cursos: Viviana Merola (ISTRAD-Sevilla), Ma Eivor Jordá (UEM- Valencia) y José Ramón Trujillo (UAM-UCM).
} 
Tabla 1. Relación de competencias para profesionales expertos en AD y SpS

(basada en Díaz Cintas, 2007a: 51-57)

\begin{tabular}{|c|c|}
\hline \multirow{4}{*}{$\begin{array}{l}\text { Competencias } \\
\text { lingüísticas }\end{array}$} & L1- Conocimiento exhaustivo del idioma materno en todas sus dimensiones \\
\hline & L2- Creatividad y sensibilidad lingüística \\
\hline & L3- Cotejo, revisión y edición de textos en lengua propia \\
\hline & L4- Conocimientos de lengua inglesa \\
\hline \multirow{7}{*}{$\begin{array}{l}\text { Competencias } \\
\text { temáticas }\end{array}$} & T1- Conocimiento general de la discapacidad y la accesibilidad \\
\hline & $\begin{array}{l}\text { T2- Conocimiento exhaustivo del mundo de la sordera y de la discapacidad auditiva así como } \\
\text { de la ceguera y de la discapacidad visual }\end{array}$ \\
\hline & T3- Conocimiento del lenguaje cinematográfico y la semiótica de la imagen \\
\hline & T4- Conocimiento de otras modalidades de accesibilidad \\
\hline & T5- Conocimiento del mercado laboral y la legislación sobre AD/SpS \\
\hline & T6- Conocimiento exhaustivo de la teoría y la práctica de la AD y SpS \\
\hline & T7- Conocimientos de Teatro e Historia del arte (AD) \\
\hline \multirow{4}{*}{$\begin{array}{l}\text { Competencias } \\
\text { informáticas } \\
\text { y aplicadas }\end{array}$} & I1- Conocimiento y manejo de ordenadores, de programas informáticos generales y de Internet \\
\hline & I2- Dominio de estrategias documentación \\
\hline & I3- Conocimiento y manejo de programas de $\mathrm{AD} / \mathrm{SpS}$ \\
\hline & I4 Conocimientos de locución AD \\
\hline \multirow{9}{*}{$\begin{array}{c}\text { Competencias } \\
\text { personales }\end{array}$} & P1- Amplia cultura general \\
\hline & P2- Capacidad de aprendizaje autónomo \\
\hline & P3-Capacidad de análisis y síntesis, y de interpretación de la información \\
\hline & P4- Capacidad de pensar en el momento, de relacionar ideas y de reaccionar con rapidez \\
\hline & $\begin{array}{l}\text { P5- Capacidad de organización, planificación, gestión de la información y de proyectos } \\
\text { profesionales }\end{array}$ \\
\hline & $\begin{array}{l}\text { P6- Capacidad de razonar de manera crítica en la resolución de problemas y la toma de } \\
\text { decisiones }\end{array}$ \\
\hline & P7- Flexibilidad laboral y capacidad de trabajar en condiciones de estrés y presión temporal \\
\hline & P8- Buena disposición para trabajar en grupo \\
\hline & P9- Capacidad de intermediación experta en entornos multiculturales \\
\hline
\end{tabular}

Fuente: elaboración propia 
centes y en el currículo del curso de especialización analizados. Cabe señalar que, aunque, en ocasiones, la denominación no coincidía exactamente, asimilamos los contenidos a las competencias descritas. Asimismo, a veces, la adquisición de una competencia no se contempla de forma expresa, pero puede deducirse de la metodología docente, del programa, de los contenidos y/o del sistema de evaluación de cada asignatura. Codificamos los datos para determinar las competencias que se citaban y la frecuencia de su presencia en las guías. Aunque el análisis que facilitamos es eminentemente cualitativo, también presentamos datos cuantitativos para completar los resultados.

\section{RESULTADOS}

\subsection{Oferta actual de estudios sobre accesibilidad en España}

Durante el curso 2018-2019, en nuestro país, no existe ningún curso de posgrado oficial que verse exclusivamente sobre accesibilidad audiovisual. Sin embargo, se oferta un título de experto en accesibilidad que la aborda con profundidad. Es el caso del Curso de Experto en Accesibilidad a la Comunicación y a los Contenidos Audiovisuales del Instituto Superior de Estudios Lingüísticos y Traducción de Sevilla (ISTRAD).

No obstante, las asignaturas sobre accesibilidad están presentes en todos los cursos de posgrado ofertados en España que abordan la TAV, lo que da cuenta de que se considera una parte fundamental de esta. Solo existen dos másteres oficiales con la denominación «traducción audiovisual» en su título. Se trata del Máster Universitario en Traducción Audiovisual de la Universidad Autónoma de Barcelona (UAB) y el Máster Universitario en Traducción Audiovisual y Localización de la Universidad Autónoma de Madrid y la Universidad Complutense de Madrid (UAMUCM). Asimismo, en cuanto a títulos propios, la Universidad de Cádiz oferta el Máster en Traducción Audiovisual: Localización, Subtitulación y Doblaje organizado por el ISTRAD y el Instituto de Lingüística Aplicada (ILA) de la Universidad de Cádiz. También, la Universidad Europea de Madrid (UEM) ofrece el Máster en Traducción Audiovisual (sede en Valencia) y el Máster en Doblaje, Traducción y Subtitulación (sede en Madrid), aunque este último está más orientado hacia la comunicación audiovisual y no tanto hacia la traducción propiamente dicha.

Tabla 2. Posgrados sobre TAV que abordan la accesibilidad curso 2018-2019

Posgrados sobre TAV que incluyen asignaturas sobre accesibilidad

$\mathrm{UAB}$

UAM y UCM

ISTRAD e ILA

UEM Madrid

UEM Valencia
Máster Universitario en Traducción Audiovisual

Máster Universitario en Traducción Audiovisual y Localización

Máster en Traducción Audiovisual: Localización, Subtitulación y Doblaje

Máster en Doblaje, Traducción y Subtitulación

Máster en Traducción Audiovisual 
152 Por último, existe otro bloque de cursos de posgrado en los que se pueden encontrar algunas asignaturas sobre accesibilidad audiovisual. Se trata de programas de posgrado que versan sobre traducción general o especializada. En general, estos másteres ofrecen la posibilidad de cursar bloques de asignaturas de especialización, en los que suelen estar presentes el doblaje y la subtitulación interlingüística, así como AD y SpS: es el caso del Máster de Comunicación Internacional, Traducción e Interpretación (Universidad Pablo de Olavide), el de Traducción Especializada (Universitat de Vic, Universitat Central de Catalunya y Universidad San Jorge), el Máster Universitario en Estudios de Traducción (Universitat Pompeu Fabra), el Máster de Traducción creativa y Humanistica (Universitat de València, Estudi General), el Máster Universitario en Traducción profesional de la Universidad de Granada o el Máster Universitario en Traducción Profesional y Mediación Intercultural de la Universidad de Las Palmas.

A toda esta oferta de posgrados universitarios, se añade ahora el Curso de especialización en $A D$ y Subtitulación cuyo currículo ha sido recientemente aprobado por el Real Decreto 94/2019, de 1 de marzo, por el que se establece el Curso de especialización en audiodescripción y subtitulación y se fijan los aspectos básicos del currículo. A pesar de estar en vigor, las distintas comunidades autónomas de España deberán desarrollar e implementar el citado curso, que incluye expresamente en su denominación las dos modalidades de accesibilidad más conocidas y que más demanda generan: la AD y la SpS, a diferencia de la mayoría de los cursos de posgrado, como hemos visto. Esto puede explicarse por la voluntad del gobierno español de formar rápidamente expertos especializados exclusivamente en accesibilidad, y no en TAV, como se viene haciendo hasta el momento en las universidades. El curso de formación profesional sobre accesibilidad no contempla ninguna modalidad «tradicional» de TAV en su currículo (doblaje o subtitulación). Como ya hemos adelantado, la formación se enmarca en los estudios de formación profesional de imagen y sonido y los perfiles profesionales descritos en el citado decreto son los siguientes:

- Guionista de proyectos de AD de obras audiovisuales y espectáculos en vivo y eventos.

- Subtitulador/a de obras audiovisuales y espectáculos en vivo y eventos.

- Locutor/a de proyectos de AD de obras audiovisuales y espectáculos en vivo y eventos.

- Revisor/a de guiones de AD.

- Revisor/a de subtítulos (art. 7.2).

Según el trabajo de Sanz-Moreno (2018: 128), nueve de los catorce audiodescriptores que participaron en aquel estudio exploratorio compaginaban su trabajo como especialistas en accesibilidad con otras modalidades de TAV más conocidas, como doblaje, subtitulación interlingüística o voces superpuestas, ya que, dada la situación del mercado laboral de la accesibilidad, no suelen encontrarse traductores que se dediquen en exclusiva a la AD o la SpS. Las personas que, hasta ahora, se vienen encargado de la accesibilidad audiovisual, realizaban otros tipos de encargos de traducción ya que el mercado no permitía una dedicación profesional en exclusiva a la accesibilidad. No obstante, el curso de especialización aprobado por el gobierno solo aborda la $\mathrm{AD}$ y SpS intralingüística, por lo que parece que los especialistas en accesibilidad se encargarán únicamente de estas dos submodalidades de TAV. En otras palabras, parece que se pretende huir del traductor multitarea que se encuentra con frecuencia en el mercado de la traducción (audiovisual), algo a lo que ya apuntaban Utray, Pereira y Orero (2009: 255): «the more tasks that can be accomplished by one 
person the faster - and cheaper — the work will be», y se forme personal con alto grado de especialización en accesibilidad.

\subsection{Requisitos de acceso}

De forma general, no es indispensable tener un grado en Traducción e Interpretación para cursar los estudios de posgrado analizados, aunque en muchos se otorga prioridad a los egresados en esta materia.

Para poder acceder al Curso de Experto en Accesibilidad a la Comunicación y a los Contenidos Audiovisuales del ISTRAD-Sevilla, el único requisito que se exige es estar en posesión de título universitario de grado o similar, por lo que, en principio, no se exige titulación en Traducción e Interpretación (aunque, en general, el perfil de los alumnos matriculados es de traducción e interpretación).

En los cursos de posgrado sobre TAV, tampoco se exige formación previa (de grado) en traducción, aunque se otorga prioridad en el acceso a los egresados de Traducción e Interpretación. En la UAB, también pueden cursar este máster los graduados en Filología, Estudios de Asia Oriental, Humanidades, Ciencias de la comunicación o titulaciones equivalentes. En la UAM-UCM se establecen prioridades de acceso: primero, los graduados y licenciados en Traducción e Interpretación, y titulaciones equivalentes, con buenos conocimientos de informática; en segundo lugar, los graduados y licenciados en Lenguas Modernas o Estudios Ingleses (con conocimientos de informática y de traducción) y, por último, los graduados y licenciados en Informática, Ingenierías y titulaciones equivalentes con buenos conocimientos de traducción. En el caso del ISTRAD-ILA, solo se requiere tener estudios superiores, aunque no se especifica la especialidad. Y en la UEM-Valencia, se requiere estar en posesión de un título de grado (con prioridad por el de Traducción e Interpretación) y haber cursado TAV en el grado o haber hecho el TFG sobre TAV.

En cuanto a los másteres generales sobre traducción que contienen alguna(s) asignatura(s) sobre accesibilidad, tienen prioridad a la hora de ingresar los graduados o licenciados en Traducción e Interpretación, aunque no se exige el título en esta materia. También se citan otros grados afines como Filología, Lenguas Aplicadas, Lenguas Modernas (UV, UVic, Las Palmas, UGR) o simplemente estudios superiores (UPF y Pablo de Olavide). En algunos casos, se valora la experiencia profesional acreditada en el ámbito de la traducción para acceder a estos estudios de posgrado (UV y UGR), independientemente de la formación de origen.

La no exigencia de un título de grado o licenciatura en Traducción e Interpretación parece corroborar lo observado en el estudio de SanzMoreno (íbid.) en el que, a pesar de que la mayoría de las profesionales de la $\mathrm{AD}$ en España provenían de estudios de Traducción e interpretación, lo cierto es que estos no consideraban esa formación esencial para el desempeño de la $\mathrm{AD}$ (y, por tanto, de la accesibilidad). No obstante, una formación previa en traducción otorga al futuro experto en accesibilidad un bagaje que le será de utilidad, así como la adquisición de competencias indispensables también para el correcto desempeño de su función (conocimiento exhaustivo de la lengua materna en todas sus dimensiones, manejo de programas informáticos de TAV, capacidad de análisis y síntesis, estrategias de documentación, cultura general, por citar solo algunos ejemplos).

Mención aparte merecen los requisitos de acceso al curso de especialización del grado superior en accesibilidad. Se puede acceder mediante el curso de Técnico Superior en Realización de Proyectos Audiovisuales y Espectáculos (Real decreto $1680 / 2011$, de 18 de noviembre) y el curso de 
Tabla 3. Requisitos de acceso y admisión a posgrados sobre TAV que abordan la accesibilidad curso 2018-2019 y al curso de especialización sobre accesibilidad

Requisitos de acceso para cursar posgrados que abordan la accesibilidad y el curso de especialización en accesibilidad

ISTRAD Sevilla

UAB

UAM y UCM
Estar en posesión de título universitario de grado o similar

Estar en posesión de un título universitario oficial

Requisitos de admisión: los licenciados o graduados en Traducción e Interpretación, Filología, Estudios de Asia Oriental, Humanidades, Ciencias de la comunicación o titulaciones equivalentes.

Estar en posesión de título universitario oficial Requisitos de admisión: los licenciados o graduados en Traducción e Interpretación, y titulaciones equivalentes; en Lenguas Modernas o Estudios Ingleses; y, por último, en Informática, Ingenierías y titulaciones equivalentes con buenos conocimientos de traducción

\begin{tabular}{ll}
\hline ISTRAD e ILA & Estudios superiores \\
\hline UEM Valencia & $\begin{array}{l}\text { Estar en posesión de un título de grado (con prioridad por el de Traducción e } \\
\text { Interpretación) y haber cursado TAV en el grado o haber hecho el TFG sobre TAV }\end{array}$ \\
\hline $\begin{array}{l}\text { Curso de especializa- } \\
\text { ción del grado superior } \\
\text { sobre accesibilidad }\end{array}$ & $\begin{array}{l}\text { Haber cursado el curso de Técnico Superior en Realización de Proyectos Audiovi- } \\
\text { suales y Espectáculos (Real decreto 1680/2011, de 18 de noviembre) o el curso de } \\
\text { Técnico Superior en Producción de Audiovisuales y Espectáculos (Real decreto } \\
\text { 1681/2011 de 18 de noviembre). }\end{array}$ \\
\hline
\end{tabular}

Técnico Superior en Producción de Audiovisuales y Espectáculos (Real decreto 1681/2011 de 18 de noviembre). Si bien es cierto que en estos títulos se hace mención del doblaje y la subtitulación, no se abordan desde su dimensión creativa, sino más bien desde un punto de vista más técnico.

\subsection{Modalidades de impartición}

El curso del ISTRAD se imparte en línea (con la opción de asistir a algunas clases presenciales), mientras que tanto el máster de la UAB como el del ISTRAD-ILA se imparten en modalidad presencial o a distancia, a elección del alumnado. Los másteres sobre TAV de la UAM-UCM y el de la UEM son solo presenciales. Los cursos de posgrado sobre traducción general también lo son.

En general, las personas que cursan posgrados sobre TAV o que se quieren especializar en accesibilidad ya disponen de ciertos conocimientos sobre traducción, y es frecuente que necesiten compaginar los estudios con el ejercicio profesional, por lo que los cursos en línea les permiten estudiar y trabajar al mismo tiempo, y además aportar una visión más profesionalizante a las enseñanzas más teóricas.

Todas las clases del curso de especialización del grado superior son presenciales, sin que el decreto contemple la posibilidad de impartir las clases de modo virtual por el momento. 


\subsection{Asignaturas}

De forma general, se identifican dos grupos de asignaturas que versan sobre accesibilidad: un primer grupo en el que se han incluido aquellas que son específicas sobre accesibilidad y que se denominan como tal («accesibilidad a los medios de comunicación», «subtitulación para sordos», «audiodescripción para ciegos», etc.).Y un segundo grupo de asignaturas sobre TAV que incluyen algunas nociones teórico-prácticas de $\mathrm{AD}$ y SpS.

En el caso de los estudios especializados sobre accesibilidad y, de forma general, sobre TAV, las asignaturas sobre $\mathrm{AD}$ y $\mathrm{SpS}$ son obligatorias. El que se impartan ambas submodalidades juntas parece lógico ya que, como indicaba Díaz Cintas, la formación en accesibilidad debería permitir «la capacitación de profesionales expertos tanto en $\mathrm{SpS}$ como $\mathrm{AD}$, lo que vendría a ser el epítome del “accesibilitador” ( (2007b: 52). Parece, pues, que el profesional debería ser capaz de realizar ambas actividades y saber adaptarse a la naturaleza de los distintos productos. La formación que se oferta parece orientada hacia la consecución de este fin.

Tabla 4. Asignaturas obligatorias de los posgrados sobre accesibilidad y TAV

\begin{tabular}{|c|c|c|}
\hline Título de posgrado & ECTS & Asignaturas \\
\hline $\begin{array}{l}\text { Experto en accesibilidad a la comuni- } \\
\text { cación y a los contenidos culturales } \\
\text { (ISTRAD) }\end{array}$ & 60 & $\begin{array}{l}\text { Módulo I: Introducción a la accesibilidad } \\
\text { Módulo II: Accesibilidad a la información y comunicación } \\
\text { Módulo III y IV: Accesibilidad a los productos audiovisuales I } \\
\text { (Subtitulación para personas con discapacidad auditiva y } \\
\text { Subtitulación para personas con discapacidad cognitiva); } \\
\text { Accesibilidad a los productos audiovisuales II (Audiodes- } \\
\text { cripción de productos multimedia y Audiodescripción en } \\
\text { artes escénicas) } \\
\text { Módulo V: Accesibilidad a los bienes culturales y al patrimonio } \\
\text { Módulo VI: Accesibilidad a los productos digitales } \\
\text { Módulo VII: Proyecto práctico }\end{array}$ \\
\hline $\begin{array}{l}\text { Máster Universitario en Traducción } \\
\text { Audiovisual (UAB) }\end{array}$ & 9 & $\begin{array}{l}\text { Una asignatura obligatoria: } \\
\text { Subtitulado para Sordos y Audiodescripción para ciegos }\end{array}$ \\
\hline $\begin{array}{l}\text { Máster Universitario en Traducción } \\
\text { Audiovisual y Localización } \\
\text { (UAM-UCM) }\end{array}$ & 6 & $\begin{array}{l}\text { Una asignatura del módulo optativo II Itinerario } 1 \text { (TAV): } \\
\text { Traducción audiovisual y accesibilidad para Personas } \\
\text { con Discapacidad Auditiva o Visual }\end{array}$ \\
\hline $\begin{array}{l}\text { Máster en Traducción Audiovisual: } \\
\text { Localización, Subtitulación y Doblaje } \\
\text { (ISTRAD-ILA) }\end{array}$ & 12 & $\begin{array}{l}\text { Dos asignaturas obligatorias: } \\
\text { Técnicas de subtitulación para deficientes auditivos } \\
\text { y personas sordas. } \\
\text { Técnicas de audiodescripción para invidentes. }\end{array}$ \\
\hline $\begin{array}{l}\text { Máster en Traducción Audiovisual } \\
\text { (UEM-Valencia) }\end{array}$ & -- & $\begin{array}{l}\text { Dos asignaturas obligatorias }{ }^{4}: \\
\text { Subtitulación para personas sordas } \\
\text { Audiodescripción }\end{array}$ \\
\hline
\end{tabular}

4 Ambas asignaturas se imparten con una duración de 72 horas. 
156 En cuanto a la presencia de asignaturas de accesibilidad en otros posgrados españoles es más residual y, en general, forma parte de asignaturas sobre TAV. Las asignaturas que se ofertan en estos estudios de posgrado son optativas y oscilan entre tres (Universidad Pablo de Olavide) y doce créditos (UVic). En cualquier caso, la optatividad proviene de la especialidad de traducción elegida (se elige entre traducción audiovisual o comunicación audiovisual, jurídica y financiera, literaria, tecnologías de la información, etc.). Pero si el alumno decide cursar la especialización en $\mathrm{TAV}$, entonces obligatoriamente estudiará la(s) asignatura(s) sobre accesibilidad. En dos universidades (UPF y Las Palmas) la accesibilidad se enseña como parte de una asignatura de TAV, mientras que en el resto la accesibilidad ya tiene entidad propia (UPO, UV, UGR, UVic). En tres casos se habla de «traducción y accesibilidad», donde se incluyen AD y SpS (no se especifica el tiempo que se dedica a cada una de las submodalidades), mientras que solo en el caso de la UVic se distinguen dos asignaturas de $\mathrm{AD}$ y dos de SpS, dando un lugar específico a cada una de ellas. En general, se trata de asignaturas teóricoprácticas, en las que se evalúa desde la redacción de un guion de $\mathrm{AD}$, el uso de herramientas para crear SpS, prácticas de $\mathrm{AD}$ según diferentes géneros, etc. El contenido teórico es importante en todas ellas, y proporcionan una base sólida sobre la que asentar la práctica de la accesibilidad, a la que se prioriza en la evaluación.

Tabla 5. Asignaturas sobre accesibilidad de los posgrados generales de traducción

\begin{tabular}{lcc}
\multicolumn{1}{c}{ Título de posgrado } & ECTS & \multicolumn{1}{c}{ Asignaturas } \\
\hline $\begin{array}{l}\text { Máster Universitario en Comunicación } \\
\text { Internacional,Traducción e Interpreta- } \\
\text { ción (Pablo de Olavide) }\end{array}$ & 3 & $\begin{array}{l}\text { Una asignatura optativa: } \\
\text { Accesibilidad a los medios }\end{array}$ \\
\hline $\begin{array}{l}\text { Máster Universitario en Estudios } \\
\text { de Traducción (U. Pompeu Fabra) }\end{array}$ & 5 & $\begin{array}{l}\text { Una asignatura optativa: } \\
\text { Traducción audiovisual }\end{array}$ \\
\hline $\begin{array}{l}\text { Máster Universitario en Traducción } \\
\text { Creativay Humanistica (UV) }\end{array}$ & 5 & $\begin{array}{l}\text { Una asignatura optativa: } \\
\text { Traducción audiovisual y accesibilidad }\end{array}$ \\
\hline $\begin{array}{l}\text { Máster en Traducción Profesional (UGR) } \\
\text { Máster Universitario en Traducción }\end{array}$ & 6 & $\begin{array}{l}\text { Una asignatura optativa: } \\
\text { Traducción y Accesibilidad }\end{array}$ \\
$\begin{array}{l}\text { Especializada (UVic, Universitat Central } \\
\text { de Catalunya-Universidad San Jorge) }\end{array}$ & 12 & $\begin{array}{l}\text { Cuatro asignaturas optativas en la especialidad } \\
\text { Comunicación audiovisual: } \\
\text { Subtitulación para Personas Sordas B-A (Inglés-Catalán) } \\
\text { Subtitulación para Personas Sordas B-A (Inglés-Español) } \\
\text { Audiodescripción para Invidentes B-A (Inglés-Catalán) } \\
\text { Audiodescripción para Invidentes B-A (Inglés-Español) }\end{array}$ \\
\hline $\begin{array}{l}\text { Máster universitario en Traducción } \\
\text { (Universidad Las Palmas de Gran } \\
\text { Canaria) }\end{array}$ & 6 & $\begin{array}{l}\text { Una asignatura optativa: } \\
\text { Traducción de textos audiovisuales }\end{array}$ \\
\hline
\end{tabular}


Tabla 6. Asignaturas sobre accesibilidad del curso de especialización en AD y Subtitulado

Curso

ECTS

30

Curso de especialización en $A D$

y Subtitulado
Asignaturas

Cuatro módulos obligatorios:

- $\mathrm{AD}$ de obras audiovisuales, eventos y espectáculos en vivo

- Subtitulación de obras audiovisuales, eventos y espectáculos en vivo

- Locución, autoría y audionavegación

- Formación en centro de trabajo

En cuanto al curso de especialización en AD y SpS del grado superior, encontramos un módulo profesional que versa exclusivamente sobre $\mathrm{AD}$ de obras audiovisuales, eventos y espectáculos en vivo, de 9 créditos; otro sobre Subtitulación de obras audiovisuales, eventos y espectáculos en vivo, de 8 créditos; y el último sobre Locución, autoría y audionavegación de 4 créditos. El resto de la formación son prácticas en centros especializados ( 9 créditos).

\subsection{Competencias}

$\mathrm{Al}$ analizar las diferentes guías docentes de las asignaturas sobre accesibilidad, observamos una gran variedad en las mismas: algunas presentan un nivel de detalle y precisión muy elevado, mientras que otras son más generales. También hemos de tener en cuenta que, dado que la accesibilidad se estudia en el marco de asignaturas sobre TAV, las competencias que se describen en estas guías suelen ser más generales y no específicas de accesibilidad audiovisual. Por último, señalamos que en el caso de los dos cursos del ISTRAD y el de la UEM, no existen guías docentes con enumeración de competencias determinadas al tratarse de títulos propios, aunque, en estos casos, hemos podido deducirlas de la información proporcionada por los planes de estudio, páginas web y programas de las distintas asignaturas, para cuya obtención hemos contactado con los coordinadores de los cursos. Lo mismo ocurre con el curso de especialización aprobado por el Real Decreto 94/2019. En este caso, hemos acudido al currículo en el que se especifican las asignaturas que se van a cursar $y$, en particular, al artículo 6, que establece las competencias específicas (profesionales, personales y sociales) que se pretende que adquieran los alumnos.

\subsubsection{Competencias lingüísticas}

Respecto a las competencias lingüísticas, tan solo tres guías docentes (UPF, UGR, UV) de las siete analizadas citan el conocimiento exhaustivo del idioma materno (L1) como competencia que debe desarrollarse, mientras que el conocimiento del inglés (L4) solo aparece nombrado en dos guías docentes (UPF, UGR). Probablemente, en estos dos últimos casos, el conocimiento de la lengua inglesa se cita porque la accesibilidad se enmarca en una asignatura más general sobre TAV, y, en el caso de doblaje y subtitulación, el inglés suele ser la lengua origen que se estudia con más frecuencia. Además, el conocimiento del castellano (o del catalán en el caso de másteres que se imparten en Cataluña, cuando se eligen las asignaturas en esta lengua) y del inglés son 
158 requisitos necesarios para acceder a los estudios de posgrado analizados. En la UPF se solicita un «nivel alto» de castellano, mientras que en la UAM-UCM, en la UPO, en la UV, en la UPalmas y en la UGR se requiere la acreditación de nivel $\mathrm{C} 1$ de conocimiento de lengua castellana (se entiende que se dispone de estos conocimientos si se ha cursado un grado universitario en España); en la UVic se exige un nivel C2 de la lengua A (castellano o catalán); la UAB es la que exige un mayor nivel de conocimiento de la lengua española ya que los estudiantes extranjeros deben acreditar un nivel C2.2. en español. El curso de grado superior no cita el conocimiento de la lengua materna o de otro idioma como competencia a desarrollar. Parece que, en este caso, no se otorga la importancia que consideramos merece la correcta redacción en español, algo que viene determinado por la norma AENOR UNE 153020. En efecto, recordemos que para la redacción de guiones audiodescriptivos en castellano, la norma exige que «deben seguirse las normas gramaticales establecidas por la Real Academia Española de la Lengua» o también que «deben utilizarse adjetivos concretos, evitando los de significado impreciso» (AENOR, 2005: 7-8).

Por lo que respecta a los conocimientos de inglés (L2), es un requisito para acceder a los cursos de posgrado de la UAB, la UAM-UCM, de la UV, de la UPalmas, de la UPO, la UGR, la UVic y de la UEM en los que debe acreditarse un nivel $\mathrm{C} 1$ o equivalente. En el ISTRAD no se exige dominio de lengua extranjera. Hace más de diez años, Díaz Cintas (2006: 20) consideraba el conocimiento del inglés como un prerrequisito incluso en la formación del experto en accesibilidad dada la importancia de esta lengua en el mercado y que, por aquel entonces, la traducción de guiones de AD del inglés al español se planteaba como una posibilidad que se podría explorar. No obstante, esta tendencia no se ha consolidado en nuestro mercado. Tampoco los participantes en el estudio de Sanz-Moreno (2018: 133) consideraron el conocimiento de la lengua inglesa como una competencia esencial en la formación del experto en accesibilidad. Consideramos que la competencia en lengua inglesa se requiere en los estudios de posgrado, de forma general, porque la accesibilidad se imparte con otras asignaturas de TAV en las que el inglés es la lengua origen y, por tanto, su conocimiento es fundamental para la realización de encargos de doblaje o subtitulación. Prueba de ello es que en el curso que versa exclusivamente sobre accesibilidad, no se requiere el conocimiento del inglés, puesto que tanto en SpS como en $\mathrm{AD}$ no se da una traducción interlingüística ${ }^{5}$.

Sin embargo, todas las guías docentes analizadas, así como el currículo del curso de especialización en accesibilidad ${ }^{6}$, nombran expresamente la competencia L3, Cotejo, revisión y edición de textos en lengua propia. La revisión del trabajo realizado se presenta como una competencia indispensable en la formación del experto en accesibilidad. La revisión del trabajo propio y del de compañeros, así como llevar a cabo un control de calidad de los productos que se hacen accesibles es la competencia lingüísti-

5 Por el momento, y dado que en España el doblaje se emplea de forma general más que la subtitulación, la $\mathrm{AD}$ se realiza en español (en la misma lengua en la que se escucha la banda sonora). No obstante, la traducción de guiones de $\mathrm{AD}$ podría constituir una interesante herramienta didáctica en la formación de futuros expertos en accesibilidad.

6 El texto remite a la verificación de la adecuación del texto del guion audiodescriptivo a la obra audiovisual o espectáculo en vivo, para asegurar la comprensión de esta por personas ciegas, pero no incide en la revisión lingüística propiamente dicha. También se hace hincapié en supervisar y aplicar procedimientos de gestión de calidad, de accesibilidad audiovisual y «diseño para todas las personas», aunque, como puede apreciarse, la redacción resulta muy general. Será necesario comprobar cómo se concretan estas competencias en el desarrollo autonómico del currículo. 
ca que aparece con más frecuencia en las guías analizadas, puede que sea porque se trata de una competencia transversal que debe desarrollarse en todas las modalidades de traducción. Asimismo, destacamos que la gestión de calidad de los productos accesibles se prevé como una de las competencias que debe reunir el experto en accesibilidad que curse el grado superior analizado. Esto supone un gran avance respecto a la situación actual. Si bien Vázquez confirmaba la existencia de un sistema de supervisión y corrección interno de las AD de la ONCE (2006: 1), lo cierto es que con la liberalización del mercado de la accesibilidad y el fin del monopolio de la producción de AD por la ONCE, se desconoce si en determinados operadores que ofrecen accesibilidad se realiza una supervisión de los controles de calidad internos. Según el estudio de SanzMoreno (2018), los audiodescriptores desconocen en la mayoría de los casos se si realiza una revisión o control de calidad de los guiones de $\mathrm{AD}$ que entregan por encargo.

\subsubsection{Competencias temáticas}

Por otra parte, en cuanto a las competencias temáticas, el conocimiento del mercado laboral y la legislación sobre $\mathrm{AD} / \mathrm{SpS}$ (T5) y el conocimiento exhaustivo de la teoría y la práctica de la $\mathrm{AD}$ y $\mathrm{SpS}$ (T6) son las que aparecen con más frecuencia mencionadas en las guías analizadas, cada una por cinco estudios de posgrado, algo lógico si tenemos en cuenta que ambas disciplinas están reguladas por normas de AENOR y que las asignaturas son teórico-prácticas. Las competencias temáticas más específicas para $\mathrm{AD}$, como el conocimiento de teatro y de historia (UGR) o el conocimiento del lenguaje cinematográfico y de la semiótica de la imagen (UAB, UGR) aparecen de forma muy residual. El conocimiento general de la discapacidad y de la accesibilidad aparece mencionado en cuatro guías docentes (UAB, UAM-UCM, UGR y UVic) y en la UEM se le dedica una sesión introductoria, mientras que el conocimiento exhaustivo del mundo de la sordera y de la discapacidad auditiva, así como de la ceguera y de la discapacidad visual solo se cita por la UAB, aunque de la lectura de las guías docentes parece que se hace referencia a esta competencia en los temas introductorios. El conocimiento de otras modalidades de accesibilidad no se cita por ninguna guía docente, quizá porque se suelen estudiar la AD y la SpS juntas. Es decir, se entiende que el «accesibilitador» deberá ser experto en ambas modalidades. En el curso de especialización de grado superior no se citan estas competencias como tal, pero en los criterios de evaluación sí se incluyen el análisis de las necesidades de las personas con discapacidad visual, la valoración de las condiciones de percepción de las personas con discapacidad y el análisis de la temática de la obra audiovisual. Destacamos además que se incluyen las dos modalidades más frecuentes de accesibilidad, es decir, en productos grabados y en productos en directo.

\subsubsection{Competencias informáticas}

En cuanto a las competencias informáticas y aplicadas, todos los planes de estudio inciden en la importancia del manejo de software específico para accesibilidad. Ocho guías docentes hacen referencia al conocimiento y manejo de ordenadores, de programas informáticos generales y de Internet mientras que seis inciden en el conocimiento y manejo de programas de $\mathrm{AD} / \mathrm{SpS}$ (ISTRAD, UPO, UAB, UV, IST-UCA, UCM- UAM, UVic y UGR). El curso de especialización otorga mucha importancia al dominio de softwares sobre audiodescripción, así como a programas de conversión de formatos, programas de locución, etc. 
Por otra parte, cinco guías docentes de posgrado inciden en la importancia de desarrollar estrategias de documentación (UPO, UPF, UAB, UV y UGR). La documentación también es una competencia que está presente en el curso de grado superior de especialización en AD y SpS («elaborar la documentación sobre detalles y descripción de escenas de la obra o espectáculo audiovisual»).

Destacamos que solo dos estudios de posgrado (ISTRAD-ILA y UAM-UCM) incluyen conocimientos de grabación, locución y montaje. Esta es quizá la mayor novedad introducida por el Real Decreto 94/2019, puesto que el experto en accesibilidad también será locutor de las AD que elabore. El audiodescriptor también adquirirá competencias de locución de los guiones de AD, tanto para productos grabados como para obras en directo, lanzando la $\mathrm{AD}$ previamente grabada, lo que supone un gran cambio respecto a lo que se viene haciendo hasta ahora. Recordemos que la norma UNE 153020 que rige la audiodescripción en nuestro país (AENOR, 2005) distinguía entre las funciones de locución y de elaboración y redacción del guion de $\mathrm{AD}$. Es más, Vázquez, uno de los audiodescriptores veteranos de la ONCE, defendía que ambas funciones debían desempeñarse por profesionales distintos (2006). Díaz Cintas, sin embargo, consideraba esencial que los audiodescriptores locutaran los guiones de $\mathrm{AD}$, tal y como se viene haciendo en Reino Unido (2007b: 58). Parece pues que el nuevo curso sobre AD establecido por el Real Decreto 94/2019 implica que el audiodescriptor sea también locutor, algo que contradice lo que se estaba demandando en el mercado actual de la AD. Así, en el estudio de Sanz-Moreno, se desprende que en España:

Los audiodescriptores realizan mayoritariamente labores de redacción del guion de AD. Solo algunos, de forma excepcional, [realizan] locuciones.
En general, los participantes coinciden en que la locución debería realizarse por el audiodescriptor en casos concretos, «siempre que se cuente con la formación adecuada» (P2, P3 y P8), así como «con una voz y dicción óptimas» (P12). Están de acuerdo en que, por el momento, y dada la ausencia de conocimientos de locución de los audiodescriptores, esta la realicen especialistas, como actores de doblaje (2018: 131).

Los profesionales de la AD están de acuerdo en que, siempre que se reciba una formación específica en locución y que se disponga de medios técnicos para poder grabarlas en condiciones óptimas, sería conveniente que el descriptor también se encargara de la locución, por lo que formar en esta materia puede resultar muy beneficioso para integrarse en el mercado laboral audiovisual. Recordemos, además, que en la $\mathrm{AD}$ teatral en directo los descriptores también son los que locutan y graban las AD; caso distinto son las AD fílmicas.

\subsubsection{Competencias personales}

Las competencias personales son las que se han mencionado en más ocasiones en las distintas guías docentes. La competencia más mencionada es la capacidad de organización, planificación, gestión de la información y de proyectos profesionales, que aparece en siete guías docentes (UPO, UAB, UV, UCM-UAM, UGR, UVic y UPalmas), algo que no es de extrañar ya que todos los estudios de posgrado analizados tienen como objetivo ofrecer una formación muy práctica directamente enfocada a la incorporación en el mercado laboral, por lo que enfrentarse a proyectos de accesibilidad reales es una prioridad en estos estudios. La buena disposición para trabajar en equipo (UV, UAB, UCM-UAM, UPO, UGR, UPalmas), la capacidad de análisis y síntesis, y de interpretación de la información (UPO, 
UAB, UPF, UV, UGR e ISTRAD-UCA), y la capacidad de intermediación experta en entornos multiculturales (UPO, UV, UCM- UAM, UGR, UVic y UPalmas) son competencias que aparecen en seis guías docentes. La capacidad de razonar de manera crítica en la resolución de problemas y la toma de decisiones y la flexibilidad laboral y capacidad de trabajar en condiciones de estrés y presión temporal también se enumeran en las guías docentes, aunque de forma más residual (en cinco y cuatro guías respectivamente). Por último, a la cultura general (UCM-UAM, UV, UGR) y a la capacidad de pensar en el momento, de relacionar ideas y de reaccionar con rapidez (UPO, UCM-UAM) no se les otorga tanta importancia si tenemos en cuenta su escasa presencia en los programas de estudio analizados.

Por último, destacamos que hemos encontrado la alusión a dos competencias que no se encontraban originariamente en el listado que nos sirve de marco teórico para nuestro análisis pero que han adquirido una presencia notable en los estudios sobre accesibilidad: estas son el desempeño del trabajo atendiendo a unos principios éticos y morales (PO, UAB, UCM-UAM, UGR) y la sensibilización o conciencia social de la necesidad de la accesibilidad para todos (PO, UAB, UCM-UAM, UGR). Por otra parte, también hay cuatro guías docentes que inciden en las diferentes formas de abordar la accesibilidad audiovisual a través de la $\mathrm{AD}$ o la $\mathrm{SpS}$ en función de los distintos géneros fílmicos (UAB, UGR, UVic, UPalmas) o del producto cultural (museos, monumentos históricos, videojuegos, etc.). Es decir, parecen adivinarse submodalidades de accesibilidad en función del producto y del género que hay que hacer accesible $\mathrm{e}^{7}$

Así, por ejemplo, la asignatura de la UAB incluye la $\mathrm{AD}$ de productos infantiles; Práctica general: $\mathrm{AD}$ de contenidos humorísticos y sexuales; $\mathrm{AD}$ del principio de una película o $\mathrm{AD}$ de artes escénicas (teatro y ópera). Lo mismo ocurre con
El curso de grado superior cita la capacidad de 161 resolver problema con iniciativa y autonomía, con creatividad e innovación, así como el trabajo en equipo, y la adaptación a nuevas situaciones laborales como competencias personales que debe desarrollar el alumnado.

\section{CONCLUSIONES}

En este artículo, hemos presentado un estudio exploratorio sobre la oferta formativa en accesibilidad en España y hemos comprobado las competencias que se pretende que adquiera el alumnado que quiera convertirse en experto en accesibilidad. Como hemos visto a lo largo de estas páginas, la formación en accesibilidad es una realidad en el panorama actual de los posgrados españoles, si bien su presencia aún aparece muy vinculada a la TAV. Esto puede que empiece a cambiar cuando se implemente el Real Decreto $94 / 2019$, que establece un curso de especialización en accesibilidad orientado sobre todo a la práctica de la $\mathrm{AD}$ y de la SpS.

A pesar de ofrecer un panorama muy aproximado de la oferta formativa en accesibilidad, somos conscientes de las limitaciones que presenta nuestro estudio. Nuestra intención ha sido incluir todos los estudios de posgrado que ofertaban asignaturas sobre accesibilidad, aunque es posible que no hayamos detectado algún máster que la contemple. Por otra parte, hemos analizado las guías docentes que están publicadas, así como los programas de cada estudio, pero es necesario tener en cuenta que, a menudo, lo que se indica en la guía docente no corresponde exactamente a lo que se imparte en esa asignatura, ya que el profesorado puede introducir algunos cambios o modificar los contenidos a lo largo del curso. Por otra parte, y dado que muchas asignaturas

el curso del ISTRAD Sevilla, que distingue entre AD Teatral, fílmica, museística, etc. 
162 que ofrecen accesibilidad se enmarcan en la TAV, es probable que en ellas se desarrollen competencias que no se hayan citado expresamente en las guías docentes o se adquieran en otras asignaturas. Además, en el caso de títulos propios, no disponemos de un listado de competencias como tal, aunque hemos intentado deducir por otras fuentes de información las competencias que se pretendía adquirir. En cuanto al curso de especialización del grado superior, el Real decreto 94/2019 establece un marco bastante general que debe desarrollarse por las comunidades autónomas, $y$, aunque permite hacerse una idea bastante aproximada del perfil de experto en accesibilidad que ha trazado el gobierno, su implementación y aplicación práctica será fundamental para corroborar cuáles son las competencias a las que se otorgan más importancia.

Consideramos que la publicación del Real decreto 94/2019 por el que se establece la formación profesional del audiodescriptor y subtitulador para sordos va a suponer un revulsivo importante en estos estudios de posgrado, puesto que la aprobación de la nueva legislación favorecerá el incremento de profesionales de la accesibilidad audiovisual en el mercado laboral. Los conocimientos que se prevén proporcionar a los futuros expertos son más prácticos, muy enfocados a la integración en empresas especializadas en accesibilidad. El «accesibilitador» conocerá la práctica de ambas modalidades de accesibilidad y, entre otras, recibirá formación específica en locución, algo que, de forma general, no se contempla en los estudios de posgrado analizados. Además, cursará un número elevado de prácticas que, en ningún caso, se ofertan en los estudios analizados, por lo que es previsible que tenga un acceso tutorizado y muy directo a la realidad del trabajo del experto en accesibilidad. Sin embargo, aunque las competencias técnicas están muy presentes en el currículo, lo cierto es que el bagaje de la formación universitaria no se contempla en este curso: el dominio de la lengua materna, el conocimiento lingüístico profundo, el análisis textual de las obras audiovisuales y el conocimiento temático parece que no se van a abordar con la profundidad con la que se abordan en los estudios universitarios. Además, es necesario señalar que los estudios de posgrado se realizan después de cuatro años de estudios universitarios, lo que otorga un bagaje cultural y lingüístico y una madurez que los alumnos del curso de especialización deberían adquirir en un periodo de tiempo más corto.

En cualquier caso, será necesario esperar unos años para comprobar si el modelo formativo propuesto por el gobierno español triunfa o si, al contrario, la accesibilidad sigue asegurándose por universitarios con estudios de posgrado en España. Actualmente, parece que los estudios de posgrado analizados cubren las competencias que Díaz Cintas (2007a) enumeró desde un plano más teórico como deseables para las personas que querían convertirse en expertos en accesibilidad, y que los descriptores que se encuentran trabajando en la actualidad en España consideran indispensables para el desempeño de su función. Sería, pues, conveniente que se siguiera fomentando el diálogo constante entre instituciones formativas, investigadores, agentes del mercado audiovisual y profesionales de la accesibilidad para adecuar la oferta formativa a lo que el mercado demanda, así como para estar al corriente de los cambios que este sufre por sus contingencias intrínsecas.

\section{REFERENCIAS BIBLIOGRÁFICAS}

Aenor (2005): «Norma UNE 153020. Audiodescripción para personas con discapacidad visual. Requisitos para la audiodescripción y elaboración de audioguías», Madrid: AENOR. 
Alonso-Pérez, Rosa y Alicia Sánchez Requena (2018): «Teaching foreign languages through audiovisual translation resources: teachers' perspectives», $A p$ plied Language Learning, 28 (2), 1-24, <http://shura.shu.ac.uk/18464/>

Calduch Carme y Noa Talavan (2017): «Traducción audiovisual y aprendizaje del español como L2: el uso de la audiodescripción», Journal of Spanish Language Teaching, 4:2, 168-180. doi: 10.1080/23247797.2017.1407173

Chaume, Frederic (2003): «Teaching audiovisual translation: Some methodological proposals» en Luis Pérez González, (ed.), Speaking in Tongues: Language across Contexts and Users, Valencia: Publicacions de la Universitat de València.

Cerezo Merchán, Beatriz (2013): «La formación en Traducción Audiovisual en España: Un estudio de caso empírico-descriptivo», TRANS: Revista de Traductología, 17, 167-183.

CESyA (2019): «Aprobado el curso de especialización en Audiodescripción y Subtitulación» <https:// www.cesya.es/articulos/201903281228>

DíAZ CinTAS, Jorge (2005): «Audiovisual translation today, a question of accessibility for all», Translating Today, 4, 3-5.

Díaz Cintas, Jorge (2006): «Competencias profesionales del subtitulador y el audioaudiodescriptor», CESyA Centro Español del Subtitulado y la Audiodescripción: Madrid https://bit.ly/2K8XQL7

Díaz CinTAS, Jorge (2007a): «Por una preparación de calidad en accesibilidad audiovisual», TRANS: Revista de Traductología, 11, 45-59.

Díaz CinTAS, Jorge (2007b): «Traducción audiovisual y accesibilidad», en Catalina JimÉnez HurTado (ed.), Traducción y accesibilidad. Subtitulación para sordos y audiodescripción para ciegos: nuevas modalidades de Traducción Audiovisual, Fráncfort del Meno: Peter Lang, 9-23.

Díaz CinTAs, Jorge (2008): «Teaching and learning to subtitle in an academic environment», en Jorge DíaZ CinTAS (ed.), The Didactics of Audiovisual Translation, Ámsterdam-Filadelfia: John Benjamins, 89-103.

Díaz Cintas, Jorge, Jordi Mas López, y Pilar Orero (2006): «Reflexiones en torno a la enseñanza de la traducción audiovisual en España. Propuestas de futu- ro», en Nobel Augusto Perdu Honeyman, Miguel Ángel García Peinado; Francisco Joaquín García Marcos y Emilio Ortega Arjonilla (coords.), Inmigración, cultura y traducción: reflexiones interdisciplinares, Almería: Universidad de Almería, 560-566.

EspaÑa. Ley 51/2003, de 2 de diciembre, de igualdad de oportunidades, no discriminación y accesibilidad universal de las personas con discapacidad (LIONDAU), BOE núm. 289, de 03/12/2003.

EsPaÑA. Real Decreto 94/2019, de 1 de marzo, por el que se establece el Curso de especialización en audiodescripción y subtitulación y se fijan los aspectos básicos del currículo, BOE núm 70, de 22/03/2019.

JimÉnez Hurtado, Catalina (2007): «De imágenes a palabras: la audiodescripción como una nueva modalidad de traducción», en Gerd WoтjAK (ed.), Quo vadis Translatologie? Berlín: Frank \& Timme, 143-159.

LeRTOLA Jennifer (2015): «Subtitling in language teaching: Suggestions for language teachers », en Yves GambieR, Annamaria CAIMI y Cristina MariotTI (eds.), Subtitles and Language Learning, Berna: Peter Lang, 245-267.

Lertola Jennifer (2018): «From Translation to Audiovisual Translation in Foreign Language Learning», TRANS: Revista de Traductología 22, 185-202.

López Noguero, Fernando (2002): «El análisis de contenido como método de investigación», XXI, Revista de Educación, 4, Universidad de Huelva, 167-179.

Maszerowska, Anna, Anna Matamala y Pilar Orero, (2014): Audio Description. New perspectives illustrated, Ámsterdam: Benjamins.

Martínez Sierra, Juan José (2008): «Hacia una enseñanza más completa de la traducción audiovisual», Revista Electrónica de Estudios Filológicos, 16. https://www.um.es/tonosdigital/znum16/secciones/estudios-11-Tradaudiovisual.htm

Matamala, Anna y Toni Badia (2007): «La docencia en accesibilidad en los medios», TRANS: Revista de Traductología, 11, 61-71.

Matamala, Anna (2006): «La accesibilidad en los medios: aspectos lingüísticos y retos de formación», en Ricardo Amat y Álvaro Pérez-Ugena (eds.), Sociedad, integración y televisión en España, Madrid: Laberinto, 293-306. 
164 Matamala, Anna y Pilar Orero (2007): «Designing a Course on Audio Description and Defining the Main Competences of the Future Professional», Linguistica Antverpiensia, 6, 329-344.

Mendoza, Nuria y Anna Matamala (2018): «La enseñanza de la audiodescripción en España: panorama actual de sus prácticas de enseñanza y aprendizaje, en base a los resultados de un cuestionario », Comunicación presentada en didTRADUAB, el 20-21 junio 2018 https://ddd.uab.cat/pub/ presentacions/2018/191174/mendoza-matamaladidtrad-2018.pdf

Mendoza, Nuria y Anna Matamala (2020): «Skills and competences of audio describers in Spain», Linguistica Antverpiensia, 18, 144-165 https://lans-tts. uantwerpen.be/index.php/LANS-TTS/article/ view/508/488

NAVARRETe, Javier (1997a): «Sistema AUDESC: el arte de hablar en imágenes», Integración, 23, 70-75.

NAVARrete, Javier (1997b): «Aplicación al teatro del sistema AUDESC», Integración, 24, 26-29.

SAnZ-Moreno, Raquel (2018): «Perfil socioprofesional del audiodescriptor en España», Hikma, 17, 119143.

Talaván Noa (2011): «La influencia efectiva de los subtítulos en el aprendizaje de lenguas extranjeras: análisis de investigaciones previas», Sendebar, 22, 265-282.

TALAVÁN Noa (2013): La subtitulación en el aprendizaje de lenguas extranjeras, Barcelona: Octaedro.

Talaván, Noa y José J. Ávila-Cabrera (2015): «First insights into the combination of dubbing and subtitling as L2 didactic tools», en Yves GambieR, Annamaria CAImi y Cristina MariotTi (eds.), Subtitles and Language Learning, Berna: Peter Lang, 149-172.

Torralba, Gloria (2017): «La subtitulación como herramienta para la adquisición de léxico en lengua extranjera», en Juan José MarTínez Sierra (coord.), Fotografía de la investigación doctoral en traducción audiovisual, 2, Madrid: Bohodon Ediciones, 357-373.

TorralBa, Gloria (2018): «Aprendizaje y perfeccionamiento de lenguas: la subtitulación en el Grado en Maestro de Educación Primaria», Revista de Lenguas para Fines Especificos, 24 (2), 73-91 <http:// dx.doi.org/10.20420/rlfe.2018.235>
Utray, Francisco, Ana Pereira y Pilar Orero (2009): «The Present and Future of Audio Description and Subtitling for the Deaf and Hard of Hearing in Spain », Meta: Journal des Traducteurs, 54-3, 248263.

VÁzquez, Antonio (2006): «Comentarios al documento "Competencias profesionales del subtitulador y del audioaudiodescriptor"». CESyA: Madrid http://www.cesya.es/recursos/CESyA 